features of sepsis.Diagnosing DKA in this age group is crucial as any delay in initiating right treatment may result in significant morbidity and mortality.

\section{P77 REYE-LIKE SYNDROME AND SEVERE CARNITINE DEFICIENCY IN A BOY WITH AUTISM SPECTRUM DISORDER AND RESTRICTED DIET}

Lydia Healy*, Eimear Forbes, Jane Rice, Jane Leonard, Ellen Crushell. Temple Street Children's University Hospital, Dublin, Ireland

\subsection{6/archdischild-2019-epa.432}

Case Description A 9-year old boy presented to the Emergency Department with an acute history of vomiting, ataxia and reduced consciousness in the setting of a respiratory illness. In the two months prior he had intermittent episodes of vomiting, without headaches or visual disturbance.

On examination, he was encephalopathic (GCS 9/15), with marked hepatomegaly. Height and weight were on the $0.4^{\text {th }}$ $2^{\text {nd }}$ percentile and $9^{\text {th }}$ percentiles respectively.

$\mathrm{He}$ had a background of high-functioning autism spectrum disorder (ASD) and a long history (since aged 18 months) of rigid eating behaviour, with a diet restricted to corn-based crisps ('Rancheros'), rich-tea biscuits (2-3/day), French-fries and Coca-Cola (10\% glucose, drinking $1 \mathrm{~L} /$ day). He had recently commenced a multivitamin preparation.

Blood-work showed anaemia, elevated hepatic transaminases and creatinine kinase $(6000 \mathrm{U} / \mathrm{l})$, low albumin, hypoglycaemia (2.7 $\mathrm{mmol} / \mathrm{l})$ and deranged electrolytes. CT brain showed moderate, communicating hydrocephalus without pressure effects. An extra-ventricular drain was inserted but later removed as this was deemed to be a coincidental finding of arrested congenital hydrocephalus.

Management and Investigation $\mathrm{He}$ required intubation and ventilation for respiratory compromise (RSV infection). Given the Reye-like scenario and abnormal biochemistry, a metabolic disorder was suspected. Acylcarnitine profile showed undetectable free carnitine and amino acid levels were low, as were vitamin levels. He received carnitine supplementation and very cautious nasogastric feeding; biochemistry and neurological status improved. Extensive investigations including carnitine uptake studies did not reveal an inherited metabolic disorder.

Detailed nutritional analysis of the patient's diet revealed very low daily protein $(0.37 \mathrm{~g} / \mathrm{kg} /$ day $)$ and fat $(0.77 \mathrm{~g} / \mathrm{kg} /$ day $)$ but with normal total daily caloric intake of $1200 \mathrm{kCal}$, indicative of protein-energy-malnutrition (Kwashiorkor). He received intensive inpatient dietetic and psychological input and by discharge from hospital he was consuming an expanded range of foodstuffs. Carnitine is synthesised from essential amino acids lysine and methionine, but primarily it is obtained directly from protein in the diet. Cereal-based diets such as above are particularly low in the amino acids methionine and lysine.

Diagnosis and Follow-up On follow-up 2 years later, carnitine levels remain normal without supplementation. Protein intake is normal and diet is varied. Vertical growth has improved significantly with height now on the $91^{\text {st }}$ centile and hepatomegaly has resolved. The final diagnosis was dietary proteinenergy-malnutrition with profound secondary carnitine deficiency resulting in Reye-like syndrome.

This case reminds us that severe malnutrition exists in the developed world, and emphasises the need for detailed nutritional assessment of children on self-restricted diets, especially those with ASD.

\section{P78 AN UNUSUAL CAUSE OF AN ACUTE CONFUSIONAL STATE IN AN ADOLESCENT; TRANSIENT GLOBAL AMNESIA}

Alwyn Charles*, Ronan Callanan, Shozab Taj, Eoin Fitzgerald, Elizabeth O'Mahony, AnnMarie Murphy. Department of Paediatrics, UHL, Limerick, Ireland

\subsection{6/archdischild-2019-epa.433}

Background Transient global amnesia (TGA) is a syndrome that has been known to occur predominantly within the adult population. It's an entity characterised by sudden loss of memory with confusion that lasts less than 24 hours. The event itself is never associated with focal neurological findings and the patient remains conscious throughout the event. Some of the precipitants documented within the literature have included swimming, immersion in cold water, extreme physical exertion or emotional stress. There has been much debate to date over the etiology/pathogenesis of TGA, including migraine, epilepsy, emboli, ischaemia of hippocampal regions or even venous congestion have all been suggested causes. While TGA is more prevalent amongst those $>60$ years of age, there has been very few instances of TGA in childhood.

Aim To describe a case of transient global amnesia in a teenage boy.

Methods We report the clinical presentation, results of investigations and outcome to date in a fourteen year old boy who presented in an acute confusional state, the ultimate cause for which was transient global amnesia.

Results A 14 year old boy presented to the Paediatric Emergency Department (PED) for evaluation of acute confusion following a hurling match. A previously well, neurodevelopmentally appropriate child, he had no significant family history nor did he have any regular medications or allergies.

Being an active sportsman, he had just participated in an intense hurling match that had lasted almost two hours in exceptionally cold weather. On cessation of the game, he had approached his coach disoriented and claiming ' $\mathrm{I}$ do not know where I am or why I am here'.

He continued to ask bizarre repetitive questions, and had no memory of the match for the following 12 hours. His neurological exam was normal, with no loss of consciousness or head injury. Basic blood work, toxicology screen, CXR and ECG were normal. Twenty fours later he had completely recovered. CT brain, MR brain with angiography and EEG were normal.

At follow-up six weeks later, he had remained perfectly well with normal neurological assessment.

Conclusion Childhood transient global amnesia is rare. Recurrence has been reported in those with a history of recurrent migraine. Our case is interesting in that it deviates from such reports whereby this episode seems solely provoked by Valsalva-like activity (extreme exertion)

\section{P79 KAWASAKI DISEASE}

Zachary Tan*, Irina Chistol, Samira Mohamed, Salma Mohamed, Nurrasyidah Abdul Halim, Victor Morris. St Luke's Hospital, Kilkenny, Ireland

\subsection{6/archdischild-2019-epa.434}

Introduction Kawasaki Disease (KD) is a relatively rare disease with a reported current, annual incidence in Europe of about $5-10 / 100,000$ children younger than 5 years_. The highest 
incidence of KD in Europe is in Ireland. Between 1996 and 2000 , the incidence rate increased up to 15.2 per 100,000 children younger than 5 years. Kawasaki Disease is known to be common in Japan and in Asian population and there is no clear understanding on aetiology of the condition.

Case Presentation We present a case of a 6 years and 4month-old boy with 5 days of pyrexia $\left(39^{\circ} \mathrm{C}\right)$, erythematous maculo-papular skin rash, joint pain, 1 day of red eyes, erythema of palms and soles. He was lethargic, irritable with reduced oral intake. He was born pre-mature at 36 weeks gestation and has history of asthma. He had no allergies. Regular medications were Montelukast and Salbutamol MDI prn. His immunisations were up to date. On examination he had temperature of $40.3^{\circ} \mathrm{C}$, heart rate 122 per minute, irritable, maculo-papular rash on knee and abdomen, red lips, strawberry tongue, erythema of palms and soles and non-purulent conjunctivitis .His investigations included elevated CRP of 72, ESR 13 mm-1 hour with normal Full Blood Counts, Urea \&Electrolytes, LFTs, ASOT and negative blood culture, urine culture, throat swab culture, and viral PCR. His serology for CMV, EBV and Mycoplasma pneumoniae were also negative. In addition, he had a negative autoimmune screen. He was diagnosed with Kawasaki Disease based on clinical criteria as per the AHA guidelines for diagnosis of $\mathrm{KD}^{2}$. He received treatment with aspirin and intravenous immunoglobulin (IVIG) infusion. He remained afebrile for 30 hours after IVIG but then developed pyrexia $\left(39.2^{\circ} \mathrm{C}\right)$ and received a second dose of IVIG as per AHA guidelines. He was transferred to Cardiology in the tertiary hospital for Echocardiogram. He remained well thereafter and was discharged home on aspirin. His follow up echocardiograms were normal. He did not develop any coronary artery aneurysms.

Discussion The case illustrates the importance of prompt diagnosis of Kawasaki to prevent complications of coronary artery dilatation, aneurysm and thrombosis leading to death. Kawasaki Disease is no longer a rare condition but an under diagnosed one. Early recognition and management of Kawasaki Disease can lead to complete recovery, whereas misdiagnosis or late diagnosis will lead to increased morbidity and mortality. With this case we hope to increase awareness of Kawasaki disease amongst Healthcare professionals especially General practitioners \& Paediatricians.

\section{P80 CASE REPORT: ALLERGY TO MILK PROTEINS}

${ }^{1}$ Irina Moldovan, ${ }^{2}$ Ecaterina Stasii". ${ }^{1}$ USMF, Chisinau, Moldova, Republic of; ${ }^{2}$ Department of Pediatrics, State University of Medicine and Pharmasy 'Nicolae Testemitanu', Chişinău, Moldova, Republic of

10.1136/archdischild-2019-epa.435

Background Cow's milk protein allergy(CMPA) is an abnormal response by the body's immune system to milk and products containing milk. It's one of the most common food allergies in children.Cow's milk protein allergy occurs in about $7 \%$ of babies who have formula milk, but in only about $0.5 \%$ of exclusively breast-fed babies.

Case presentation The patient is a 2 years old boy. He was the product of a normal, full-term pregnancy and weighed 3,0 $\mathrm{kg}$ at birth. From 2 till 6 months of age, being on exclusive breastfeeding, the boy periodic presents loose stools, weight lossand abdominal distension with excessive flatulence within 2 hours after feeding. Sometimes it was associated with vomiting but without fever. Each episode of diarreia was caused by ingestion of cow`s milk by his mother. The rest of the history was unremarkable except for one episode of idiopathicurticaria at his mother and allergic rhinitis at hisgrandmother. Significant findings on physical examination revealed a malnourished childpale with sparse, fluffy hair, with no oedema. Systemic examination was normal. The immunologic data showed the hyper IgE-emia (1300 IU/L). The significant increase of the specific IgE: to cow`s milk protein -65 , to $\alpha$-lactalbumin 45 and to $\beta$-lactoglobulin-36, to casein 21, to bovine serum albumin - 26. Cow's milk and other milkcontained foods were excluded from the mother's and boy's diet.Elimination of identified triggers from the diet had significant positive impact in clinical recovery. Howevere this restricted diet was shortened by parents and the recurrence of clinical signs appeared. At the age of 13 months the boy had developedangioedemawhen he ate a milk chocolate and at 18 months he have suffered the first episode of urticaria when he consumedbisquit whit milk powder. At the age of 2 years the boywas referred to the emergency unit with acute urticaria. Mother have denied the fact, that any milk contained product was given to child. However, after detailed discussion mother have indicated, thatthe diet of the boyhave included the meat of the rabbit which had lactation after given birth.

Conclusion Food allergy, especially CMPA, should be considered more frequently and the milk free diet should be prescribed and explained to mothers and caregivers carefully. Cow's milk is the usual cause of milk allergy, but milk from sheep, goats, buffalo and other mammals such rabbits also can cause a reaction.

\section{P81 PSEUDO-BARTTER SYNDROME AS INITIAL PRESENTATION OF CYSTIC FIBROSIS IN A 4-MONTH OLD INFANT}

Elena Georgieva*, Nadja Rimpova, Mihail Zeljaskov, Mariana Ardalieva, Daniel lliev. University Childrens Hospital, Sofia, Bulgaria

\subsection{6/archdischild-2019-epa.436}

Cystic fibrosis (CF) is the most common autosomal recessive disease in Northern Europe. Manifestations of CF are heterogeneous and vary in severity, depending on the organs affected. Chronic lung disease, sinopulmonary ivolvement, pancreatic insufficiency and increased sweat electrolyte concentration are typical findings. Electrolyte and acid base disturbances are unusual at presentation. Genetic predisposition and febrile episodes with dehydration are among the facilitating factors for the occurrence of these disturbances.Hypocholoremic alkalosis with hyponatremic dehydration are thought to be the most common ones. The constellation is referred to as pseudo-Bartter syndrome.

A 4-month-old female infant was admitted to the ICU with poor feeding, recurrent vomiting, diarrhea and failure to thrive. Laboratory findings revealed severe metabolic alkalosis and hypokalemia, low serum sodium and chloride levels. Elecrolyte disturbances showed hyponatremic dehydration with severe hypokalemia: $\mathrm{Na}^{+} 122 \mathrm{mmol} / \mathrm{l}, \mathrm{K}^{+}$2,4 mmol/l, $\mathrm{Cl}^{-} 57 \mathrm{mmol} / \mathrm{l}$. Biochemical analysis is consistent with renal failure. Upon physical examination the child was crying weakly, had signs of mild to moderate dehydration and body temperature was normal. On lung auscultations there were no pathological findings.Inflammatory markers were raised and there was evidence of anemia. Liver function and 